\title{
OBSERVATORIO
}

\section{NUEVOS MEDIOS, NUEVO ECOSISTEMA}

\section{New media, new ecosystem}

\section{João Canavilhas}

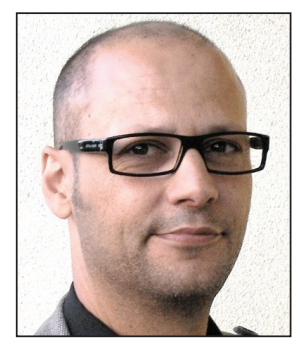

João Canavilhas es licenciado en comunicação social por la Universidade da Beira Interior (UBI, Portugal) y doctor por la Universidad de Salamanca (España). Es vicerrector en la UBI y profesor de periodismo. Su trabajo de investigación se centra en el campo de la comunicación y nuevas tecnologías, particularmente en la influencia de internet y de los dispositivos móviles en el periodismo. Es autor o coautor de 6 libros, 24 capítulos de libro y 32 artículos en revistas científicas nacionales e internacionales. Participa en proyectos de investigación en Portugal, España y Brasil. http://orcid.org/0000-0002-2394-5264

Universidade da Beira Interior (Covilhã) Av. Marquês d’Ávila e Bolama, 6200-001 Covilhã, Portugal jc@ubi.pt

\section{Resumen}

En las últimas décadas, los avances tecnológicos han permitido llevar las noticias más lejos, más rápido y a más consumidores. Las limitaciones espaciales y temporales, que siempre han afectado al sector de la información, perdieron su importancia, abriendo una ventana de oportunidades ofrecida por la digitalización y por la masificación de internet. Sin embargo, el potencial prometido rápidamente se desvaneció: la falta de inversión, el voluntarismo excesivo y la distancia empresas/investigación llevaron a la industria de la comunicación a sucesivos fracasos, con el cierre de miles de medios de comunicación en todo el mundo. Hoy en día, pocos dudan que el periodismo vive uno de los peores periodos de su historia. Puede parecer extraño que la evolución tecnológica haya convertido una oportunidad en una dificultad, pero ocurrió. ¿Pero cómo se puede invertir el proceso, transformando la dificultad actual en la anterior oportunidad? Se hacen propuestas para explorar las posibilidades de los dispositivos móviles y encontrar en ellos una salida para la crisis que afecta a las empresas de comunicación.

\section{Palabras clave}

Medios; Nuevos medios; Ecosistema mediático; Medios online; Móviles; Internet; Redes sociales; Modelos de negocio.

\begin{abstract}
In recent decades, technological advances have enabled the news to go farther, faster and to more consumers. The spatial and temporal constraints that had always affected the information sector lost their importance, opening a window of opportunity thanks to digitization and the internet massification. However, the promised potential quickly faded: the lack of investment, excessive voluntarism and the distance between media companies and research led the media industry to successive failures, with thousands of media closed worldwide. Today, there is no doubt that journalism is experiencing one of the worst periods in its history. It may sound strange that technological evolution has converted an opportunity into a difficulty, but it happened. Nonetheless, how can the process be reversed, transforming the current difficulty into the previous opportunity? This paper delivers some proposals that seek to explore the potential of mobile devices and looks towards these as an escape from the crisis that affects the media companies.
\end{abstract}

\section{Keywords}

Media; New media; Media ecosystem; Online media; Mobile devices; Internet; Social networks; Business models.

Canavilhas, João (2015). “Nuevos medios, nuevo ecosistema”. El profesional de la información, v. 24, n. 4, pp. $357-362$. http://dx.doi.org/10.3145/epi.2015.jul.01 


\section{Un nuevo ecosistema}

La entrada de nuevas especies en un ecosistema siempre provoca cambios en los equilibrios existentes, siendo la magnitud del cambio proporcional a la importancia de la nueva especie. En el ecosistema mediático, el nacimiento y masificación de la radio cambiaron el equilibrio existente en el primer cuarto del siglo XX, reduciendo el papel de la prensa.

Cuatro décadas más tarde la entrada de una nueva especie en el ecosistema -la televisión- dio lugar a un nuevo cambio, esta vez más profundo al tratarse de un medio potente que rápidamente ganó audiencia a la prensa y a la radio.

La masificación de la Web, a finales de los años 90 del siglo pasado, y la irrupción de los medios móviles, en los primeros años del nuevo siglo, provocaron un nuevo cambio en el ecosistema, quizás el más fuerte debido a la forma en la que va aumentando el número de usuarios. El impacto ha sido tremendo en los tres factores identificados por Canavilhas (2011a):

- intermediáticos

- tecnoambientales

- contextuales

En los factores intermediáticos, entre los que se incluyen el estudio de los medios y sus relaciones, el cambio más importante ha sido el surgimiento de dos nuevas especies -la Web y los dispositivos móviles- que modificaron los contenidos y la forma de consumir información.

Según Internet World Stats, a finales de junio de 2014 había más de 3 mil millones de usuarios, es decir, el $42,3 \%$ de la población mundial estaba conectada a internet.

http://www.internetworldstats.com/stats.htm

En el caso de los móviles, ITU (International Telecommunication Union) estima que a finales de 2014 existían 6.915 millones de conexiones en todo el mundo, el $95,5 \%$ de la población. La misma organización informa de la existencia de 2.315 millones de conexiones por internet móvil, donde se incluyen los smartphones y las tabletas, lo que representa un $32 \%$ de la población mundial.

http://www.itu.int/en/ITU-D/Statistics/Pages/stat/default. aspx

Los medios no estaban preparados para el desafío del nuevo ecosistema

Los factores tecnoambientales, todo lo que se relaciona con las interfaces, siguen cambiando a una gran velocidad, ofreciendo al usuario un lugar central en el ecosistema y acercándose cada vez más al concepto de extensiones del hombre (McLuhan, 1968). Los Ilamados wearables, como Google glass, son los ejemplos más cercanos de cómo las interfaces van evolucionando y cambiando la forma de ubicarnos en el nuevo ecosistema. Blogs y redes sociales ya habían creado las condiciones para que el usuario se acercara al centro del ecosistema y, en la actualidad, es en estos ambientes en los que pasamos una parte importante del tiempo y donde consumimos información. Datos de
PEW de marzo de 2014 (Matsa; Mitchel, 2014) indicaban que el $30 \%$ de los americanos recibía noticias vía Facebook, una red que según Statista tiene 1.350 millones de usuarios en todo el mundo.

http://www.journalism.org/2014/03/26/8-key-takeawaysabout-social-media-and-news

http://www.statista.com/statistics/272014/global-socialnetworks-ranked-by-number-of-users

Entre los factores contextuales relacionados con los ambientes de consumo, los cambios son igualmente importantes por la movilidad de los receptores, lo que obliga al emisor a preparar los mensajes para varios entornos. Los nuevos medios y sus interfaces están atrayendo nuevos públicos que no consumían noticias y aumentando el tiempo de consumo como consecuencia de internet móvil y sus dispositivos de acceso.

Este cambio en el ecosistema podría resultar una oportunidad para los medios de comunicación, pero "la combinación de una revolución tecnológica, de nuevas (y todavía no entendidas) reglas de negocio, y la recesión global, han creado, para usar un cliché, una 'tormenta perfecta'”' (Jukes, 2013).

Si por un lado se vive una crisis económica global que afecta al sector de la comunicación, por otra parte hay todo un mundo de oportunidades disponible para las empresas preparadas para explorar este nuevo ecosistema mediático. Para que esto sea posible hay que responder a obstáculos situados en tres campos:

- Profesional: el desafío está en la preparación de periodistas con capacidades para actuar en un nuevo ecosistema. No se trata de cambiar los principios profesionales enunciados por Kovach y Rosenstiel (2007), si no de tener profesionales preparados para trabajar con nuevos lenguajes multimediáticos y en ambientes menos controlados, donde los usuarios también forman parte del sistema;

- Tecnológico: el nuevo ecosistema envuelve nuevas rutinas de producción, donde conceptos como proximidad o periodicidad exigen nuevas interpretaciones a la luz de las tecnologías emergentes;

- Económico: los modelos tradicionales de ingresos, ventas y publicidad no son suficientes para viabilizar los medios. Los usuarios rechazan los medios tradicionales y buscan la información en los medios online, que suelen ser gratuitos. Pero la información gratuita no tiene la calidad exigida por los usuarios y para mejorarla, los medios necesitarían disponer de unos ingresos que los usuarios no quieren satisfacer porque siempre encuentran algún tipo de información gratis en algún lugar de internet.

Estamos pues ante una serie de obstáculos, un conjunto de desafíos a los que hay que responder lo más rápidamente posible. Este trabajo es una contribución a la discusión del tema.

\section{Desafíos profesionales}

La reducción de plantillas, la competencia de los medios online gratuitos y la emergencia de los agregadores de información presionan a los medios de comunicación tradicionales para producir mayor cantidad de información de forma más rápida y con menos recursos. Sin tiempo para salir a la calle a recoger información, contactar con sus fuentes y 
confirmar las informaciones, el periodismo se trasforma en churnalism $[$ churn (inglés) $=$ batir, revolver] (Davies, 2008, p. 73).

Los medios no estaban preparados para el desafío del nuevo ecosistema. A pesar de que algunos factores fueran externos-como la crisis económica- la ausencia de recursos humanos preparados para el mundo online ha sido fatal. La respuesta a las nuevas exigencias ha tardado demasiado y el producto final, los contenidos informativos, ha perdido calidad hasta el punto de no diferenciarse de los contenidos gratuitos.

Así, el primer y más urgente desafío profesional es preparar periodistas multiplataforma y multimedia (Salaverría; García-Avilés; Masip, 2007) para trabajar en ambientes online (Tejedor-Calvo; Corpus; Lozano, 2011). La mejor forma de atraer a los lectores es hacer un producto diferente de lo que se ofrece gratuitamente, lo que implica formar periodistas que dominen nuevos lenguajes. $Y$ como no es lo mismo trabajar en medios controlados, con distribución localizada y públicos relativamente homogéneos, que trabajar en un medio global, es igualmente importante preparar periodistas con capacidad para entender la Red en toda su dimensión.

\section{El primer y más urgente desafío profe-} sional es preparar periodistas multiplataforma y multimedia para trabajar en ambientes online

Otra característica de la Web es el constante surgimiento de nuevas herramientas: trabajar con los foros, espacios de comentarios, blogs, repositorios multimedia, o las redes sociales supone todo un conjunto de conocimientos que exigen profesionales preparados para nuevas funciones. Entre otras muchas:

- search engine optimization (SEO) (Dick, 2011);

- blogger influenciador;

- news moderator.

- dosificador de información (Flores-Vivar, 2008);

- coordinador multimedia;

- editor web;

- community manager (Aguado-Terrón; Palomo-Torres, 2010).

Un segundo desafío profesional es la integración de los usuarios en el sistema mediático (Heinonen, 2011; Domin- go et al., 2008). Los comentarios pueden ser una importante fuente de información, pero es necesario que los profesionales entiendan los espacios de comentarios como una participación ciudadana que puede contribuir a mejorar la calidad del periodismo.

Un tercer desafío profesional está conectado con la pos-producción de contenidos digitales. La posibilidad de cambiar textos después de su publicación, de recoger información en las redes sociales, o de manipular imágenes digitales exige una nueva ética digital (Canavilhas, 2011b), es decir, un conjunto de reglas estables que ayuden a los periodistas a trabajar en un ambiente en que la evolución es rápida, con nuevas herramientas que crean nuevas cuestiones deontológicas.

\section{Desafíos tecnológicos}

Parte de los desafíos profesionales emergen de los cambios tecnológicos en el periodismo en los últimos años. De un ambiente en el que las fronteras entre medios se presentaban perfectamente definidas se pasó a otro donde los conceptos estabilizados durante décadas se difuminan por la emergencia de internet y de los dispositivos móviles.

Las nuevas tecnologías reclaman nuevas formas de producir, presentar y distribuir la información porque su influencia se hace sentir en todas las fases del proceso periodístico. Con el $42 \%$ de la población mundial conectada a internet, el 32\% conectada con banda ancha móvil y la previsión de que en 2020 los dispositivos móviles sean la principal forma de acceder a internet (Rainie; Anderson, 2008), parece claro que el futuro del periodismo está en este tipo de dispositivos. En el nuevo ecosistema mediático se imponen las tecnologías en la producción, presentación y distribución de información facilitando enfoques transmediáticos (Westlund, 2013) y cambiando múltiples aspectos en las fases más importantes del proceso noticioso.

En el campo de la distribución, después de décadas en que las personas iban a por la información, entramos en una era en la que la información tiene que ir a por las personas. La información tiene que estar donde se encuentran 


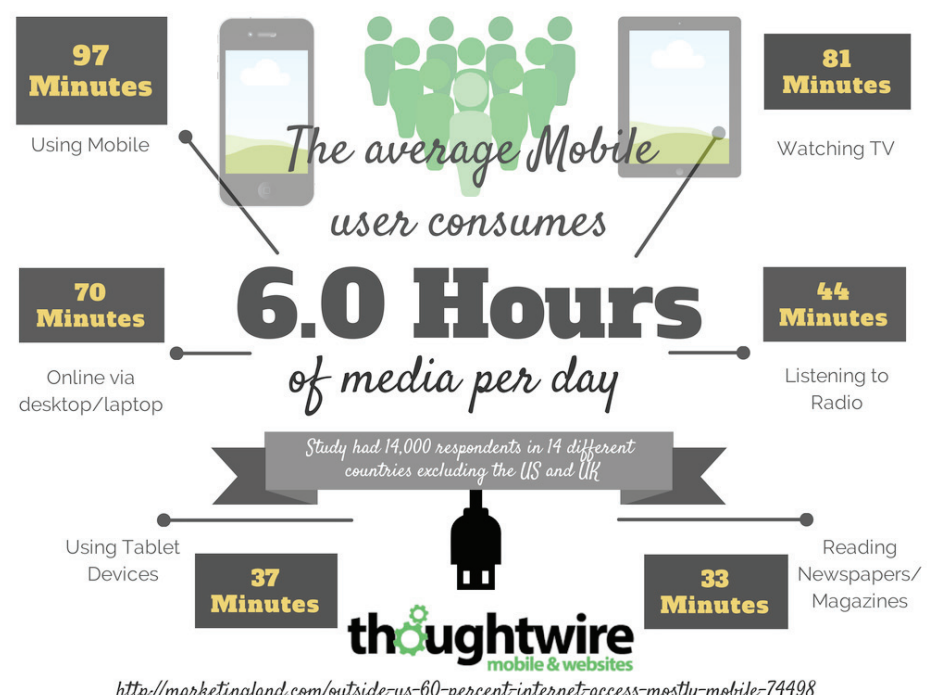

http://marketingland.com/outside-us-60-percent internet-access-mostly-mobile-74498

http://marketingland.com/outside-us-60-percent-internet-access-mostly-mobile-74498

En este último caso hablamos también de la necesidad del tercer nivel de desafío tecnológico, éste más conectado con la recepción: la emergencia de los dispositivos móviles hace que el consumo cambie y pase de ser grupal y estático a individual y móvil. Desde los tiempos de los clubs de lectura, donde la persona más alfabetizada leía el periódico a un grupo, y aquellos en los que la radio y la televisión ocupaban el lugar central en la sala familiar, se ha pasado a una era en la que la información se consume individualmente en cualquier lugar desde un smartphone o una tableta. Esto hace que la información deje de ser discutida y contextualizada por algún familiar o amigo, lo que implica más responsabilidad para los medios, retomando su misión de formar e informar.

Además, los usuarios ya no son consumidores pasivos y aspiran a un lugar central en el sistema. La nueva audiencia activa quiere espacios

las personas y en actualidad las personas están permanentemente conectadas desde su PC, smartphone o tableta. La distribución tiene que pasar de un sistema pull a un sistema push (Fidalgo; Canavilhas, 2009), manteniendo a los usuarios informados a cada momento. Esto implica igualmente un cambio en otra marca del periodismo tradicional: la periodicidad. Los informativos con horarios establecidos o la hora de salida del periódico no pertenecen a un mundo en el que la información circula a una gran velocidad. La nueva marca del periodismo tiene que ser la instantaneidad, el continuum informativo (Barbosa, 2013) que transmite la idea de una conexión permanente al mundo, que es una de las razones por la que los usuarios adquieren móviles (Katz; Aakhus, 2002). De lo contrario, los medios perderán lectores que irán a otro tipo de plataformas informativas automáticas cuya gran ventaja es mantener este flujo permanente de información, aunque ésta sea poco estructurada y superficial.

\section{Después de décadas en que las personas iban a por la información, entramos en una era en que la información tiene que ir a por las personas}

Esta necesidad de diferenciación con relación a los motores de búsqueda, y otros tipos de sistemas automáticos, es esencial para que los usuarios se presten a pagar por la información online. Por ello, los contenidos son otro desafío tecnológico. De los tradicionales contenidos monomedia replicados en la Web, los medios de comunicación deben pasar a la producción de contenidos multimedia interactivos que sepan explorar las características únicas de la Web, involucren al usuario y estén estructurados por niveles de información. De esta forma, los usuarios que prefieren noticias cortas se mantienen informados, y los usuarios más exigentes puedan satisfacer sus necesidades de contextualización con una información más profunda que se transforma en un valor añadido. de interacción con los contenidos, lo que implica igualmente cambios en el sistema.

\section{Desafíos económicos}

Las cuestiones económicas son quizá las que más están condicionando la evolución de los nuevos medios. Pero también de los tradicionales, sobre todo la prensa. El mercado de consumo sigue decreciendo porque las generaciones intermedias compran menos periódicos y las nuevas presumen de informarse en internet. Pero los espacios donde buscan informarse no acaban de identificar modelos económicos que les permitan mantener el negocio en internet. Los medios han acumulado los prejuicios de todos los cambios ocurridos en el ecosistema, pero no saben cómo recaudar ingresos con las nuevas oportunidades generadas por internet y los móviles (Grueskin; Seave; Graves, 2011)

El informe Mediascope Europe (IAB, 2012), que estudió 28 países europeos, concluyó que el $65 \%$ de la población se conecta a internet regularmente, de la cual un $90 \%$ accede a sitios de noticias. El estudio revela igualmente que el $21 \%$ accede a internet desde su móvil y el $48 \%$ usa internet mientas ve la televisión, lo que revela un nuevo fenómeno: la segunda pantalla. Esta tendencia se viene acentuando en los últimos años y confirma que los usuarios necesitan complementar la información que reciben en la televisión.

Otro informe de Mediascope, de 2013 (IAB, 2013) sobre los jóvenes (16-24 años), muestra que el $89 \%$ se conecta a internet, un valor que se acerca ya a la televisión (92\%) y sobrepasa a la radio (59\%), los periódicos (52\%) y las revistas (46\%). El tiempo pasado en internet por los jóvenes es un $30 \%$ superior a la media de la población europea y el consumo de los medios tradicionales en las plataformas digitales está subiendo. El fenómeno de la segunda pantalla presenta igualmente valores impresionantes, con un $62 \%$ de los jóvenes confirmando que ven la tele y en simultáneo están conectados a internet desde su ordenador $(86 \%)$, smartphone (43\%), tableta (13\%) o consola de juegos (15\%). 
En este escenario los medios tienen que buscar modelos económicos que saquen partido de las tendencias de consumo, porque estos jóvenes nativos digitales serán los consumidores de información en los próximos años.

En primer lugar, hay que explorar el potencial de los nuevos dispositivos móviles. Al tratarse de aparatos personales con una gran incorporación de tecnología (capacidad multimedia, GPS, acelerómetro, etc.), se abre un amplio campo de posibilidades de personalización de la información y la publicidad.

En segundo lugar, percibir la conexión permanente de los usuarios como un canal invariablemente abierto. Al asumir que las noticias son productos perecederos y, como tales, pierden valor a lo largo del día, se requiere una política de diferenciación de precios. La información más actual es más valorada, pero a lo largo del día pierde valor, por lo que su precio debe disminuir. Como alternativa, la información puede actualizarse para que mantenga el valor.

\section{Los informativos con horarios estableci- dos o la hora de salida del periódico no pertenecen a un mundo en el que la in- formación circula a gran velocidad}

En tercer lugar, los nuevos medios tienen que estudiar formas de ofrecer a los usuarios la posibilidad de interactuar con sus periodistas, de participar en foros internos, de crear lazos con el medio. Se trata de implementar estrategias de lealtad basadas en la valoración de una red propia que puedan involucrar al usuario, creando sentimientos de pertenencia al medio.

Por último, los medios tienen que buscar nuevas formas de generar ingresos que mejoren los tradicionales modelos de ventas e ingresos publicitarios y los armonicen con las nuevas oportunidades. Los modelos de 360 grados tienen como objetivo ampliar la base de posibles ingresos, buscando en las economías de escala, en la diversidad y en la proximidad al usuario, formas de identificar fuentes alternativas de ingresos. En este caso se propone una combinación de siete elementos:

- Optar por un modelo freemium, con oferta de algunas noticias y acceso condicionado a micropagos en otras. El rasgo está en la implementación de un sistema que permita la compra por unidad informativa de una forma simple (estilo iTunes).

- Tener una distribución de noticias multiplataforma y multicanal para responder a una audiencia móvil que a lo largo del día empieza por consumir información en este dispositivo, durante la jornada lo hace en su ordenador y por la noche en la tableta.

- Buscar nuevas formas de publicidad más inmersivas y que involucren al usuario. Juegos y realidad aumentada son buenas opciones.

- Con un espacio disponible casi infinito y una audiencia global, los medios online pueden dedicarse a la investi- gación en temas de nicho. Este tipo de público es sensible a acciones de crowdfunding, otro elemento para generar ingresos.

- Con el número de dispositivos móviles creciendo de forma exponencial, la demanda de aplicaciones nativas (apps) crece igualmente de forma rápida. La venta de apps es otra alternativa de financiación.

- Las empresas que ganan con los servicios informativos deben compartir costes. Los proveedores de servicios internet (internet service providers, ISP) y los fabricantes de dispositivos móviles saben que una parte importante de sus clientes adquieren servicios y equipos para, entre otras cosas, acceder a la información. Los modelos actualmente en uso -oferta de dispositivos con un período de suscripciones en periódicos u oferta de un dispositivo con la suscripción- pueden ser perfeccionados para que las empresas de comunicación encuentren formas alternativas de financiación.

La combinación de todos estos elementos, o de parte de ellos, es una alternativa para que los medios de comunicación puedan encontrar una forma de superar las dificultades sentidas en el actual ecosistema mediático.

La información a medida, es decir, la información en contexto es la forma de sacar partido de las capacidades de los medios y responder a las expectativas de los usuarios

\section{Notas finales}

El sector de la comunicación social tradicional vive probablemente los tiempos más difíciles de su historia. Con la caída de ingresos de publicidad y de ventas/suscripciones, se han recortado las plantillas, situación que impacta en la calidad del producto final. Al mismo tiempo, la evolución de internet y de los dispositivos móviles ha modificado los equilibrios en el ecosistema mediático, introduciendo nuevas variables que los medios tradicionales deberían poder controlar.

A pesar del contexto negativo, el sector tiene en estos nuevos medios la oportunidad de reciclar viejos conceptos y adaptarse a una realidad donde la posibilidad de distribuir información personalizada es un valor añadido. La información a medida, es decir, la información en contexto es hoy por hoy la forma de sacar partido de las posibilidades de los medios y responder a las expectativas de los usuarios. Ofrecer información relevante, capaz de adaptarse a las necesidades informativas de cada usuario, en cualquier lugar y en el momento más correcto es una posibilidad real.

La información tiene que estar donde están las personas y la forma de conseguirlo es distribuirla para los dispositivos que acompañan a los usuarios durante todo el día: ordenadores, móviles y tabletas. El desafío está en encontrar la forma de producir y distribuir contenidos adaptados a esta nueva realidad con calidad suficiente para que el usuario vea el pago como la consecuencia normal del servicio prestado. 


\section{Bibliografía}

Aguado-Terrón, Juan-Miguel; Palomo-Torres, Bella (2010). "Convergencia y nuevas rutinas profesionales: luces y sombras del periodista polivalente en las redacciones españolas". En: López-García, Xosé; Pereira-Fariña, Xosé. Convergencia digital: reconfiguración de los medios de comunicación en España, Santiago de Compostela: Spicusc, pp. 129-148. ISBN: 9788498873795

Barbosa, Suzana (2013). “Jornalismo convergente e continuum multimídia na quinta geração do jornalismo nas redes digitais". En: Canavilhas, João (Org.). Notícias e mobilidade: O jornalismo na era dos dispositivos móveis. Covilhã: Livros Labcom, pp. 33-54. ISBN: 9789896541026 http://www.livros/abcom.ubi.pt/book/94

Canavilhas, João (2011a). "El nuevo ecosistema mediático". Index comunicación, n. 1, pp. 13-24.

http://journals.sfu.ca/indexcomunicacion/index.php/ indexcomunicacion/article/view/4

Canavilhas, João (2011b). "Ensino do jornalismo: o digital como oportunidade". En: Quadros, Cláudia; Caetano, Kati; Laranjeira, Álvaro. Jornalismo e convergência: ensino e práticas profissionais. Covilhã: Livros Labcom, pp. 13-20. ISBN: 9789896540630

https://ubithesis.ubi.pt/handle/10400.6/717

Davies, Nick (2008). Flat earth news: An award-winning reporter exposes falsehood, distortion and propaganda in the global media. London: Random House. ISBN: 97800995 12684

Dick, Murray (2011). "Search engine optimisation in UK news production". Journalism practice, v. 5, n. 4, pp. 462-477. http://dx.doi.org/10.1080/17512786.2010.551020

Domingo, David; Quandt, Thorsten; Heinonen, Ari; Paulussen, Steve; Singer, Jane B.; Vujnovic, Marina (2008). "Participatory journalism practices in the media and beyond: an international comparative study of initiatives in online newspapers". Journalism practice, v. 2, n. 3, pp. 326-342. http://jclass.umd.edu/classes/jour698m/domingo.pdf http://dx.doi.org/10.1080/17512780802281065

Fidalgo, António; Canavilhas, João (2009). "Todos os jornais no bolso: Pensando o jornalismo na era do celular". En: Rodrigues, Carla (Org.). Jornalismo on-line: modos de fazer, pp. 99-117. ISBN: 9788520505397

http://webx.ubi.pt/ fidalgo/antonio-fidalgo-canavilhastodos-jornais-bolso.pdf

Flores-Vivar, Jesús-Miguel (2008). “Perspectivas de docencia, aprendizaje e investigación del ciberperiodismo para una emergente sociedad en red". Anàlisi, n. 36, pp. 53-63. http://ddd.uab.cat/pub/analisi/02112175n36/02112175n3 6p53.pdf

Grueskin, Bill; Seave, Ava; Graves, Lucas (2011). The story so far: what we know about the business of digital journalism. New York: Columbia University Press. ISBN: 9780231 160278 http://cjrarchive.org/img/posts/report/The_Story_So_Far.pdf

Heinonen, Ari (2011). "The journalist's relationship with users. New dimensions to conventional roles". En: Jane Singer; Hermida, Alfred; Domingo, David; Heinonen, Ari; Paulussen, Steve; Quandt, Thorsten; Reich, Zvi; Vujnovic, Marina. Participatory journalism: Guarding open gates at online newspapers, pp. 34-55. New York: Wiley-Blackwell. ISBN: 9781444332261

http://jclass.umd.edu/classes/jour698m/Singerbook.pdf http://dx.doi.org/10.1002/9781444340747.ch3

IAB (2012). Mediascope Europe. Pan-European launch presentation summary. IAB Europe.

http://goo.gl/6d5kle

IAB (2013). Mediascope Europe. The connected life of digital natives. IAB Europe.

http://goo.gl/ylECd7

Jukes, Stephen (2013). "A perfect storm". En: Fowler-Watt, Karen; Allan, Stuart (eds.). Journalism: new challenges. Centre for Journalism \& Communication Research, Bournemouth University, pp. 1-18. ISBN: 9781910042014 https://microsites.bournemouth.ac.uk/cjcr/publications/ journalism-new-challenges

Katz, James E.; Aakhus, Mark (2002). Perpetual contact: Mobile communication, private talk, public performance. Cambridge: Cambridge University Press. ISBN: 97805210 02660

Kovach, Bill; Rosenstiel, Tom (2007). The elements of journalism: What newspeople should know and the public should expect. New York: Three Rivers Press. ISBN: 9780 307346704

Matsa, Katerina-Eva; Mitchell, Amy (2014). "8 Key takeaways about social media and news". Pew Research Center. Journalism and media, March 26.

http://www.journalism.org/2014/03/26/8-key-takeawaysabout-social-media-and-news

McLuhan, Marshall (1969). Pour comprendre les média. Paris: Ed. HMH Letée.

Rainie, Lee; Anderson, Janna (2008). "The future of the internet III". PEW Research Center. Internet, science and tech, December, 14.

http://www.pewinternet.org/2008/12/14/the-future-ofthe-internet-iii

Salaverría, Ramón; García-Avilés, José-Alberto; Masip, Pere (2007). Convergencia periodística: propuesta de definición teórica y operativa. Documento de trabajo, Proyecto Convergencia digital en los medios de comunicación. http://www.ae-ic.org/santiago2008/contents/pdf/ comunicaciones/134.pdf

Tejedor-Calvo, Santiago; Corpus, Roberto-Silva; Lozano, Fernando (2011). “La formación del ciberperiodista 2.0”. En: Quadros, Cláudia; Caetano, Kati; Laranjeira, Álvaro. Jornalismo e convergência: ensino e práticas profissionais. Covilhã: Livros Labcom, pp. 21-42. ISBN: 9789896540630

Westlund, Oscar (2013). "Mobile news: A review and model of journalism in an age of mobile media". Digital journalism, v. 1, n. 1, pp. 6-26.

http://dx.doi.org/10.1080/21670811.2012.740273 